\title{
Corela
}

Cognition, représentation, langage

12-1 | 2014

Vol. $12, \mathrm{n}^{\circ} 1$

\section{Temporalité et aspectualité en anglais : opérations, représentations, cognition}

\section{Gilbert Ghio}

\section{(2) OpenEdition}

\section{Journals}

\section{Édition électronique}

URL : http://journals.openedition.org/corela/3468

DOI : $10.4000 /$ corela.3468

ISSN : 1638-573X

\section{Éditeur}

Cercle linguistique du Centre et de I'Ouest - CerLICO

\section{Référence électronique}

Gilbert Ghio, «Temporalité et aspectualité en anglais : opérations, représentations, cognition », Corela [En ligne], 12-1 | 2014, mis en ligne le 25 juin 2014, consulté le 30 avril 2019. URL : http:// journals.openedition.org/corela/3468; DOI : 10.4000/corela.3468

Ce document a été généré automatiquement le 30 avril 2019

\section{(c) (i) (2)(2)}

Corela - cognition, représentation, langage est mis à disposition selon les termes de la licence Creative Commons Attribution - Pas d'Utilisation Commerciale - Partage dans les Mêmes Conditions 4.0 International. 


\title{
Temporalité et aspectualité en anglais : opérations, représentations, cognition
}

\author{
Gilbert Ghio
}

\section{Introduction}

1 Nous ne pouvons appréhender le monde qu'à travers la médiation de nos sens. Nous n'avons donc accès qu'à une représentation de la réalité construite à travers nos perceptions, et si nous voulons communiquer avec autrui par le langage nous le faisons à partir de cette représentation. Le langage est donc une représentation de représentation. Le lien entre le langage et la réalité passe par la conceptualisation

«Structures at a given level arise through cognitive operations (...) performed on the structure at lower levels » (Langacker, 1991, pp. 3-4).

2 Il n'est pas alors interdit de penser que les structures grammaticales reflètent la façon dont nous concevons cette réalité

«Language structure (...) reflects patterns of human conceptualization because it is shaped by them » (Heine, 1997, p. 16)

*ghiogilbert@gmail.com

et «Les agencements de marqueurs sont traités comme le codage de représentations et

d'opérations mentales fondamentales " (Lapaire, 2006, pp. 25-53).

3 Notre conception de la réalité étant intimement liée à la perception que nous en avons il n'est pas surprenant de trouver des analogies entre les structures du domaine de la perception et les structures du domaine conceptuel censées la représenter

«Numerous aspects of construal that are quite important linguistically can reasonably be interpreted as general conceptual analogs of phenomena well-known in visual perception » (Langacker, 2000, p. 206).

Notre perception est essentiellement sensori-motrice et spatio-temporelle. De même que nous percevons les objets dans l'espace les uns par rapport aux autres, nous pouvons aussi situer les événements dans le temps les uns par rapport aux autres 
"One can observe a parallel between the coherence through time in linguistic

discourse and that in visual perception " (Talmy, 2000, pp. 90-91).

5 C'est parce que le temps, contrairement à l'espace, est dépourvu de toute matérialité et n'est qu'une construction de l'esprit (Michon, 1992) que sa représentation s'est structurée sur le modèle de la représentation spatiale (Jackendoff, 1985) :

« Factive temporality is expressed in terms of fictive spaciality ( (ibid).

6 C'est ainsi que des notions comme l'antériorité et la postériorité sont communes aux deux domaines. Ce n'est donc pas un hasard si l'expression linguistique de l'espace et celle du temps partagent parfois les mêmes expressions procédurales ou conceptuelles :

She arrived at two o'clock./the station.

J'ai dormi jusqu'à Paris/midi. J'ai attendu un temps infini.

\section{Conceptualisation}

7 Le temps sert à mesurer l'espace parcouru. ${ }^{1}$ Situer des objets dans l'espace c'est parcourir mentalement la distance qui les sépare. On définit ainsi une trajectoire qui est à la fois une représentation spatiale et temporelle. Dans le domaine du langage la conceptualisation du temps se fait également en termes de spatialisation. Sans entrer dans le détail, on peut dire que le temps est conçu métaphoriquement à partir de la représentation de l'espace, mais à la différence de celui-ci qui est tri-dimensionnel, il est unidimensionnel (Talmy, 2000).

8 Chaque famille de langues conceptualise le temps à sa façon en faisant appel à des constituants élémentaires invariants ancrés sur la perception du monde et les processus d'action sur le monde. ${ }^{2}$ La représentation conceptuelle du temps dérivée des formes linguistiques n'est pas en prise directe avec la représentation perceptuelle que nous en avons, en partie calquée sur la représentation spatiale. Il semblerait que les opérations linguistiques donnent lieu à des représentations sémantico-cognitives ou schèmes sémantico-cognitifs qui utiliseraient pour leur construction des archétypes primitifs de troisième niveau (opérations élémentaires d'identification, de différentiation, d'ouverture, de fermeture, notions d'intérieur, d'extérieur, d'antériorité, postériorité, simultanéité, déplacement,...). Ces archétypes s'ancrent dans la perception visuelle de l'espace et du mouvement à travers le repérage d'entités par rapport à des lieux et dans la perception de stabilisations ou de changements dans le temps (Desclés, 1991).

Ces invariants abstraits du langage seraient l'interface entre la représentation perceptuelle et la représentation conceptuelle dérivée de la représentation linguistique. La conceptualisation du temps a donc une double dimension: universelle, ontologique dérivée de la perception et particulière, linguistique correspondant à des cadres de références déterminés par la langue.

10 L'anglais se situe dans le cadre référentiel indo-européen qui est le cadre relatif basé sur le schéma corporel de l'observateur: ce qui est devant lui est du futur, ce qui est à l'opposé du passé. La temporalité y a une double dimension, computationnelle et représentationnelle : elle concerne à la fois la localisation du procès à l'aide de temps et de marques lexicales et sa vision à travers l'aspect grammatical combiné à l'aspect lexical du verbe (Asic, 2004).

11 Les temps de l'anglais sont au nombre de deux : le présent et le passé ou transpassé car le présent peut prendre plusieurs valeurs (A. Joly et D. O'kelly, 1991). 

d'énonciation ou autre) et à déterminer les coordonnées relatives des procès entre eux. Il serait faux de croire cependant qu'ils ne reflètent pas aussi la façon dont les procès sont conceptualisés. En effet, le présent et le prétérit simples sont des aoristes qui traduisent une vision holistique du procès et qui sont susceptibles de gommer en partie l'aspect lexical du verbe. Les procès peuvent aussi être vus de façon dynamique comme des événements (transitoires) ou des processus (en déroulement) ou bien statique comme des états (permanents). Les événements ont pour propriété d'être sécants, c'est à dire qu'ils interrompent un état initial et introduisent un nouvel état ; les processus donnent à voir une portion de procès saisie transitoirement dans son déroulement; les états, au contraire, présentent une vision statique du procès.

13 La vision du procès dépend d'abord des propriétés sémantico-cognitive du verbe mais l'aspect grammatical peut venir le souligner ou au contraire le neutraliser. Par exemple l'aspect duratif d'un verbe comme work appelle automatiquement la forme $b e+V$-ing dès lors que le moment du procès est identifié au moment d'énonciation ('I work/I'm working). Inversement, l'aspect statique d'un procès comme know sera neutralisé par la même forme au point d'être recatégorisé en processus (she is knowing a terrible experience). De même, les verbes téliques qui construisent par défaut le terme du procès (buy, build,...) et les verbes d'achèvement (find, reach,...) ne peuvent normalement se construire avec la marque de l'inaccompli ( ${ }^{*} \mathrm{He}$ 's building) sauf en cas de complémentation (He's building a house). De la même façon les verbes atéliques (snow, write,...) ne peuvent se construire avec la marque de l'accompli have+en (*I have written) sauf à être suivis d'un complément instaurant un terme (I've written two letters). On voit ainsi comment un élément lexical peut aussi influencer la représentation d'un procès. A l'aspect lexical du verbe qui est de nature ontologique se superpose donc l'aspect grammatical qui est de nature représentationnelle. La combinaison des deux détermine la représentation globale du procès (voir ci-dessous, 3). s'assimiler à une opération de fenêtrage. Une fenêtre se construit avec et par son contenu et correspond à la représentation d'une portion d'espace-temps : elle délimite l'espace dans lequel a lieu l'événement. Sa position est déterminée par l'auxiliaire, son contenu par le verbe et sa dimension par le type d'intervalle dû à la forme linguistique globale (Col et Victorri, 2007).

15 Si le sens temporel est défini par la position de la fenêtre et son contenu, la vision a cependant toujours son origine au moment d'énonciation qui est aussi le moment de conceptualisation.

\section{Représentations}

Dans les langues indo-européennes le temps est conçu de façon linéaire, comme une succession d'instants dans un parcours orienté sur lequel sont situés les événements les uns par rapport aux autres et au moment d'énonciation, frontière entre le réalisé et le non réalisé. Il est toutefois nécessaire de préciser que la position de ce dernier varie avec le temps chronologique : le présent d'énonciation se déplace continuellement sur l'axe du temps car le sujet origine des repères fait partie du référentiel temporel et ne peut s'abstraire du temps. Ce moment d'énonciation est donc en réalité un processus 
inaccompli ${ }^{3}$ et les marqueurs grammaticaux de temps ne font que fixer les positions relatives des événements par rapport à un moment d'énonciation en perpétuel mouvement (cette caractéristique aura son importance dans l'étude des différentes valeurs du présent). Comme le dit Guentchéva (2010)

"Le référentiel temporel est un système de repérage qui est un ensemble d'instants linéairement ordonné et structuré par des relations de concomitance, de différentiation par antériorité ou postériorité ».

Le moment d'énonciation (noté $T_{0}$ ) joue aussi le rôle de moment de référence (noté $T_{R}$ ), comme l'a montré (Vendler, 1957). Ce moment de référence peut aussi être distinct, ou confondu avec le moment de l'événement/du procès (noté $T_{E}$ ). Le temps verbal exprime donc un rapport entre trois coordonnées notées $T_{0}, T_{R}$ et $T_{E}$, ce qui fait, par exemple, qu'un énoncé comme *Jane arrived est irrecevable car dépourvu de repère temporel.

A côté des marqueurs grammaticaux de temps $(\emptyset,-e d)$ qui situent le procès par rapport au moment d'énonciation l'expression de la temporalité inclut des marques lexicales ( yesterday, the day after, two minutes later, etc...) qui précisent le moment du procès.

19 Il faut aussi tenir compte de l'aspect grammatical qui concerne la représentation du procès

- l'aoriste (présent et prétérit simples) qui correspond à une représentation holistique

- l'accompli (noté have+V-en) qui correspond à une représentation statique

- l'inaccompli (noté be+V-ing) qui correspond à une représentation dynamique

- lequel se combine à l'aspect lexical du verbe qui correspond à la nature du procès

- ponctuel (break, drop) : le procès est de nature instantanée

- duratif (work, snow) : le procès est de nature étendue

- itératif (tap, hop) : le procès est de nature répétitive

- télique (look, loose) : le procès est de nature terminative

- atélique (swim, drive) : le procès est de nature non terminative

- statique (be, have) : le procès est de nature permanente

ainsi que du contexte: un verbe peut changer d'aspect en présence d'un modifieur (l'adverbe everyday, par exemple, transforme un procès ponctuel comme stop en procès itératif).

Cela pose le problème de la représentation topologique d'un procès et donc de son bornage.

Les différents types de procès reflètent les différentes façons dont ils sont conceptualisés ${ }^{4}$

- les procès de type état (permanence : be, stay) : bornes gauche et droite ouvertes ] [

- les procès de type processus (déroulement) : borne gauche fermée, droite ouverte [ [

- les procès de type événement (discontinuité) : bornes gauche et droite fermées []

- les procès de type duratif/atélique (rain, write) : bornes séparées [ ]

- les procès de type ponctuel/télique (break, buy) : bornes confondues []

Les événements découpent l'espace topologique en deux parties : un état antérieur à l'événement (état initial), et un état postérieur (état final).

Pour résumer, la représentation temporelle d'un procès est caractérisée par :

- sa localisation temporelle (temps)

- sa représentation (aspect grammatical)

- sa nature (aspect lexical)

- son intervalle temporel (bornage)

- sa complémentation (marques lexicales) 

qui doit être prise en compte dans la représentation temporelle d'un énoncé.

\section{Le présent} singulier étant une survivance du passé). L'absence d'auxiliaire et de marque verbale de temps fait dire que le procès est conçu dans une relation d'indépendance par rapport au moment d'énonciation (absence de repérage), qu'il s'agit d'une simple prédication d'existence, d'où son emploi dans des énoncés de type générique. Le présent simple peut ainsi instancier des procès de nature différente : états, événements, mais aussi vérités générales, proverbes. Il renverra à des durées et des intervalles différents en fonction de la nature du verbe. A l'intérieur d'une même catégorie il peut aussi renvoyer à des valeurs multiples. Le terme « état », par exemple, peut recouvrir différentes valeurs

- localisation: he stays/lives in Paris

- propriété intrinsèque: he is tall/he has blue eyes

- propriété extrinsèque: she is nice/she likes chocolate

- possession: he has/owns a car

- identification: this is Martin

- état résultatif: the mouse has been eaten

Il parait nécessaire de distinguer entre un procès stable (this is Martin, he is tall,) c'est à dire non susceptible de variation et un procès stabilisé (he stays in Paris, she is nice,) susceptible d'être interrompu par un événement. Alors que les états stables ne peuvent pas être recatégorisés en processus (*he is being tall), c'est fréquemment le cas des états stabilisés (she is being nice, he's staying in Paris). Il semble que plus on s'éloigne de la notion prototypique d'état, plus la recatégorisation en processus est possible. C'est le cas en particulier des verbes à sujets non agentifs (like, know, cost,...) et des verbes de perception involontaire (see, hear,...)

How are you liking it here?

This house is costing us a lot of money.

He couldn't believe that he was seeing what he saw.

Au présent simple ces verbes décrivent plus un événement qu'un état

Through the blinds I hear the birds in his garden (Pinter, « Tea Party »).

I understand ( = passage de <not understand $>$ à $<$ understand $>$ ).

Inversement, il existe des verbes de processus ${ }^{5}$ qui font référence à une situation stabilisée

My wife stays at home but I work

où le verbe work décrit plus un état du sujet (I'm a working man) à opposer à stay qu'une activité. Il existe en fait un grand nombre de verbes de processus dans des énoncés décrivant une situation stable ou stabilisée

I work at a petrol station ( = I'm a petrol station attendant)

This magazine comes out every week ( = It's a weekly)

He comes from Paris ( = He is a Parisian)

C'est parce qu'avec le présent simple un verbe de processus est découplé du moment d'énonciation qu'il est en mesure de rendre ce concept de permanence que l'on qualifie d'habitude ou de propriété. C'est ce que met en lumière le recours à des paires contrastives comme 
I work at a petrol station / I'm working at a petrol station

He comes from Paris / He's coming from Paris

où le présent simple signale une situation stable ou stabilisée et la forme aspectuelle, parce que rattachée au moment d'énonciation, une activité temporaire.

Il $\mathrm{y}$ a cependant des cas où la valeur de vérité du présent simple se rapporte uniquement au moment d'énonciation: ce sont les indications scéniques, les modes d'emploi, les recettes de cuisine, les commentaires sportifs, etc. Le moment du procès est alors concomitant $^{6}$ au moment d'énonciation. C'est ce qui est mis en évidence par les paires suivantes

I break the egg / I'm breaking the egg.

Rebecca goes out / Rebecca is going out

où dans les deux cas l'événement a lieu au moment de parole de l'énonciateur et sa «durée » intrinsèque est identique. La différence est donc uniquement de nature conceptuelle et se réalise dans la langue par les formes aspectuelles appropriées. ${ }^{7}$

$C^{\prime}$ est particulièrement le cas des énoncés performatifs, où la forme aspectuelle est exclue ${ }^{8}$

I declare the meeting open / ${ }^{*}$ 'm declaring the meeting open

ou encore quand le présent du procès n'est pas concomitant avec le moment d'énonciation (présent dit « de narration », « journalistique », futur)

In 1750 Bach dies.

The Queen visits a new hospital.

I bet it snows tomorrow.

31 On ne peut plus parler dans ce cas de propriété du sujet. Il s'agit toujours d'événements ( Bach dies fait référence au fait de mourir, pas à l'état d'être mort) mais ayant une existence quasi autonome si ce n'était les marques lexicales de temps.

En l'absence totale de référence temporelle (explicite ou implicite) on accède au plus haut degré de généralisation et donc à l'autonomie. C'est le cas des vérités générales, des proverbes

The sun rises in the east.

This book reads well.

Charity begins at home.

En utilisant le présent simple l'énonciateur s'intéresse plus à la notion de procès qu'au procès lui-même comme le montre la possibilité de rendre les exemples ci-dessus par un nom en français, lorsque celui-ci existe : "Je suis pompiste ", "Visite de la Reine ", "C'est un Parisien", "C'est un bi-mensuel», etc... La possibilité d'accomplir une opération de réification avec de tels énoncés montre qu'ils renvoient non seulement à un procès stable mais aussi autonome. Au niveau conceptuel cela signifie que le procès est objectivé. L'énonciateur s'intéresse plus à la valeur de vérité du procès qu'au procès lui-même. Cela est particulièrement vrai dans les proverbes au point que la sémantèse du verbe se dilue dans le sens global de l'énoncé qui ne rend plus compte d'un événement mais d'un fait.

Il est toutefois impossible de tracer une frontière entre les différentes valeurs du présent simple. Il existe plutôt un continuum allant du particulier (procès en situation) au plus général (proverbes atemporels) en passant par des procès stabilisés ou stables 
I break the egg

I work at a petrol station

I like chocolate

He comes from Paris

Charity begins at home

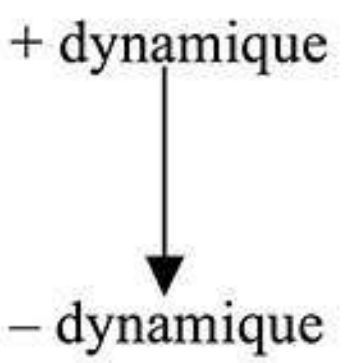

\begin{abstract}
valeur de vérité du fait correspond à un présent qui s'étire indéfiniment, par opposition à celle d'un événement concomitant avec le moment de parole (moment présent) et valable uniquement ce moment là. Lorsque le procès n'a pas de valeur de vérité au moment présent comme dans I bet it snows tomorrow, il prend une valeur épistémique et devient alors une proposition.
\end{abstract} corespond une dilatation plus ou moins grande du moment présent. La permanence de la

\section{Représentation topologique}

En l'absence de repérage, c'est la nature du procès (ponctuel/duratif) ou la marque lexicale de temps qui limite la validité du procès à la situation (ou à un moment spécifique) ou non. 
The sun rises in the east Charity begins at home This book reads well (faits) In 1750, Bach dies The Queen visits a new hospital He comes from Paris (faits)

Les procès break, understand sont de nature ponctuelle (bornes confondues). En l'absence de marque sur le verbe et de moment de référence explicite la nature du verbe et la situation font qu'ils sont concomitants au moment d'énonciation $\mathrm{T}_{0}$, comme le montre la possibilité d'ajouter now sans modifier fondamentalement le sens de l'énoncé. En présence d'un marqueur de temps explicite comme tomorrow, le procès est mentalement translaté à ce moment $\mathrm{T}_{\mathrm{E}}^{\prime}$, mais la conceptualisation a toujours son origine au moment d'énonciation (flèche verticale). En présence d'un marqueur itératif (everyday) le procès se répète suivant la fréquence indiquée. Dans le cas d'un verbe d'état (like) ou d'un verbe d'aspect duratif comme work, et en l'absence de marque lexicale du moment du procès c'est l'aspect lexical du verbe conjugué au déplacement sur l'axe du temps du moment d'énonciation qui donne sa permanence au procès : un procès duratif ou de type état est validé à chaque nouvelle position de $\mathrm{T}_{0}$ sur l'axe du temps. La vision du procès est donc prospective (dans le sens du temps chronologique).

les événements mentionnés ci-dessus ont pour point commun de ne pas être achevés : ceux concomitants avec $T_{0}\left(T_{0}\right.$ est la borne droite d'un ouvert) ainsi que les procès itératifs et les états (ils sont reproductibles). Ceux qui ont eu lieu (In 1750, Bach dies. The Queen visits a new hospital. the sun rises in the east) sortent alors du cadre référentiel car leur valeur de vérité est atemporelle, leur existence prime sur leur occurrence : ils sont devenus des faits.

\section{Le prétérit}

41 Par rapport au présent simple, le prétérit est la forme marquée. La marque -ed du prétérit signale l'antériorité du moment du procès par rapport au moment d'énonciation et l'absence d'auxiliaire sa vision holistique. C'est cette marque et l'indication lexicale de localisation du procès qui sont responsables de la structuration temporelle de l'énoncé. Le prétérit marque toujours une rupture (d'ordre temporel, modal, etc...) par rapport au moment d'énonciation. Du point de vue temporel, le moment de l'événement et le moment de référence (souvent confondus) sont distincts du moment d'énonciation et se situent à un moment antérieur à ce dernier. ${ }^{10}$

Jane arrived at/before two o'clock / yesterday / two days ago / during the holidays...

I worked at a petrol station when I was 20 / in 1980 / last month / before 1980.

L'absence d'indication explicite ou implicite du moment de l'événement (qui est aussi le moment de référence) fait que des énoncés comme *Jane arrived, *I worked... sont irrecevables. Pour permettre la localisation, le moment de l'événement doit aussi être 
précis, ce qui explique l'irrecevabilité d'énoncés comme ${ }^{*} I$ worked in the past car la référence est trop vague.

Le prétérit simple est un aoriste, c'est à dire que le procès est saisi dans sa globalité comme au présent simple et décrit des événements ou des états. Par contre le fenêtrage est différent : le procès étant révolu, distancé du moment d'énonciation il est limité à la perception d'une occurrence de procès localisée en un point précis sur l'axe du temps. Le prétérit simple gomme en partie l'aspect lexical du verbe si bien que des procès de nature et de portée temporelle différentes au présent simple se trouvent uniformisés au prétérit. Que le procès soit de type état ou événement, ponctuel, duratif ou itératif, sa nature passe au second plan et sa portée temporelle est circonscrite au moment de l'événement qui est perçu de façon identique comme un point sur l'axe du temps. ${ }^{11}$ Avec un procès itératif, par exemple, les occurrences sont globalisées et perçues comme une entité et non comme une succession de procès comme au présent simple. Cela est dû au fait que le moment du procès est fixe et distinct du moment d'énonciation alors qu'au présent simple il peut se déplacer avec lui sur l'axe chronologique du temps.

\section{Représentation topologique}

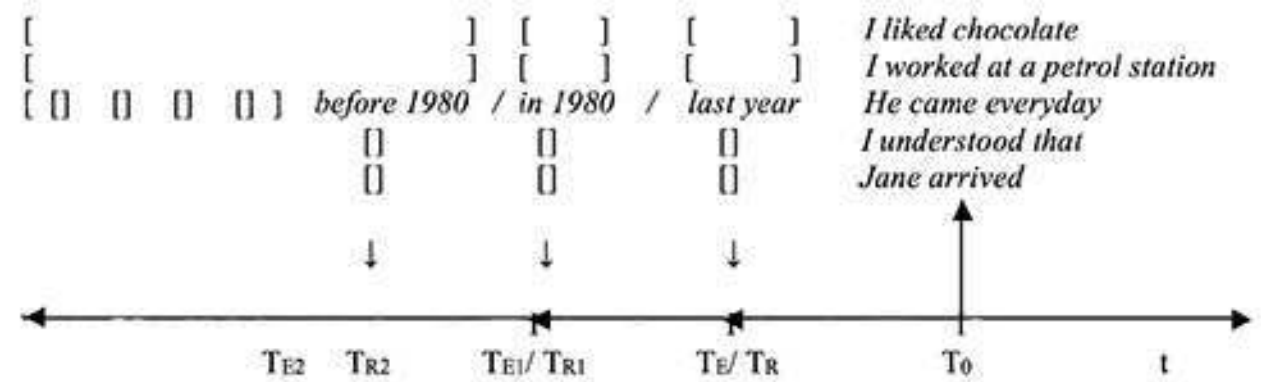

Avec le prétérit, la vision est toujours rétrospective (à l'opposé du temps chronologique). La conceptualisation se fait toujours au moment d'énonciation $T_{0}$ (flèche verticale) mais le procès est vu depuis ce moment dans un mouvement rétrospectif. En ce qui concerne les procès ponctuels (arrive, understand,...), que le moment du procès soit lui aussi ponctuel ( two o'clock) ou étendu (yesterday, last year, before 1980) n'est pas pertinent : Jane arrived last year est perçu de façon identique à Jane arrived at two o'clock ou Jane arrived before 1980. Les moments $d u$ procès sont tous perçus comme un point sur l'espace-temps, sans considération du moment précis où l'événement a pu avoir lieu. En ce qui concerne les procès duratifs (I worked at a petrol station), de type état (I liked chocolate) ou itératifs (He came everyday) l'extension à la totalité de la période n'est pas pertinente non plus pour la même raison. ${ }^{12}$

Si les différents procès font l'objet d'une représentation semblable c'est parce que, contrairement au présent simple, nous ne sommes plus dans l'actuel mais dans l'actualisé. Le procès révolu fait l'objet d'une conceptualisation sensiblement différente qui gomme les différences entre types de procès et entre moments de l'événement. D'autre part, les procès étant révolus, ils sont tous nécessairement à borne droite fermée.

Certains énoncés à double marque temporelle méritent d'être mentionnés

John (had) left a week ago yesterday (=Yesterday, John (had) left a week ago). 


\section{Représentation topologique}

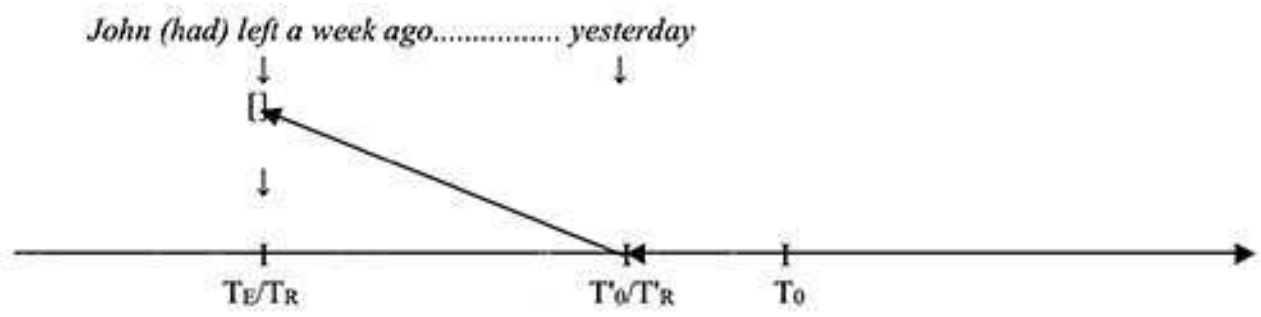

Le moment d'énonciation $T_{0}$, origine de la vision du procès, est mentalement translaté en $\mathrm{T}_{0}^{\prime}$ (yesterday), moment de référence de l'énoncé $\mathrm{T}_{\mathrm{R}}^{\prime}$ et donc de a week ago moment de référence du procès $T_{R}$ confondu avec le moment du procès $T_{E}$. On peut aussi avoir un Past Perfect à valeur d'aoriste translaté avec le même sens, ramenant le moment origine de la vision en $\mathrm{T}_{0}$.

\section{L'inaccompli}

Avec l'aspect be+V-ing, au contraire, la vision est dynamique : l'énonciateur donne à voir le procès en tant qu'activité. Pour cela il se focalise sur une portion de procès vue au moment repère. Le contenu sémantique du procès est identique, c'est la structure conceptuelle qui est différente, en relation avec des opérations de fenêtrage distinctes. Dans le cas du présent simple l'énonciateur donne à voir un objet qui par nature n'a pas besoin d'être rattaché à un moment précis pour exister. Dans le cas de la forme aspectuelle il montre un processus qui est par nature transitoire et doit donc s'inscrire obligatoirement dans la temporalité pour exister, d'où le repérage. Dans les deux cas la fenêtre s'ouvre sur le procès à partir du moment d'énonciation (la conceptualisation se faisant toujours au moment de l'élocution) mais avec la forme aspectuelle la fenêtre focalise une portion de procès saisie transitoirement au moment repère. La vision est prospective et l'intervalle associé au procès est ouvert à droite. Avec cette forme la fenêtre est limitée à la portion de procès saisie au moment repère, alors qu'avec le présent simple elle peut s'ouvrir largement sur l'infini.

\section{Représentation topologique}

L'auxiliaire be, marqueur d'identification, signale la coïncidence du procès avec le moment repère $T_{R}$, le présent que ce dernier est confondu avec le moment d'énonciation $\mathrm{T}_{0}$, la marque -ing la portion de procès considérée et les marques lexicales le moment de l'événement $T_{E} / T_{E}^{\prime}$.

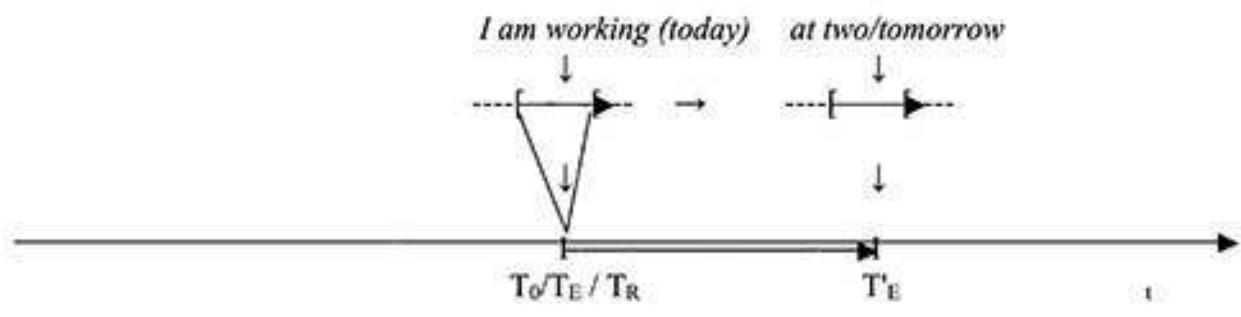




\section{Représentation topologique}

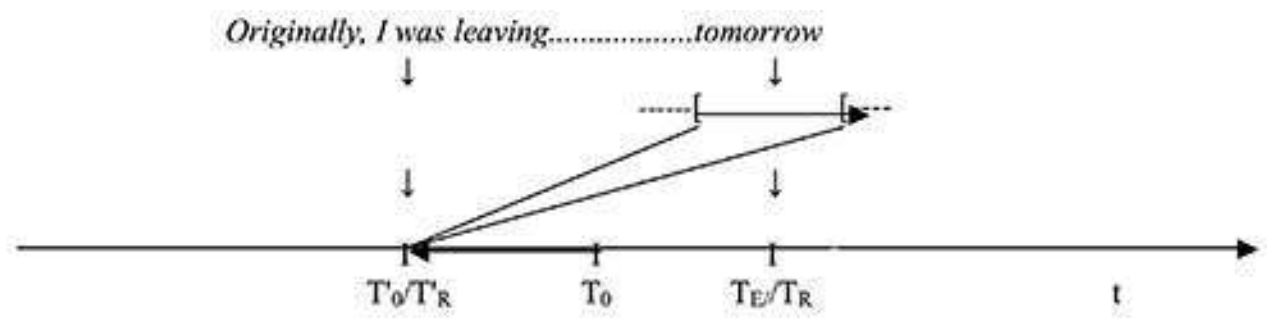


Le moment envisagé du procès $T_{E}$ est tomorrow, mais la conceptualisation du procès est translatée à son moment origine (originally) $\mathrm{T}_{0}^{\prime}$ qui devient ainsi le nouveau moment repère $T_{R}^{\prime}$ signalé par le prétérit. Be marque l'identification de la conceptualisation du procès avec le moment d'énonciation translaté $T^{\prime}{ }_{0}$ confondu avec le moment repère $T_{R}^{\prime}$. Le hiatus entre le moment repère du procès $T_{R}$ construit à partir de $T_{0}$ et le moment repère de l'énoncé $\mathrm{T}_{\mathrm{R}}^{\prime}$ construit à partir de $\mathrm{T}_{0}{ }_{0}$ (originally) fait que l'on en déduit que le procès ne sera pas validé. L'énoncé reste cependant grammaticalement acceptable car la virgule séparant originally du reste de l'énoncé montre qu'il sert de repère à tout l'énoncé, incluant tomorrow.

\section{L'accompli}

Contrairement aux cas précédents où le procès était ancré soit dans le présent soit dans le passé, la spécificité de la forme have+V-en tient au fait qu'elle participe à la fois du présent et du passé. Contrairement au prétérit où le procès est révolu (absence de repérage par rapport à un repère point de vue), avec l'accompli il n'y a pas de rupture avec le moment repère (moment d'énonciation pour le Present Perfect ou moment passé pour le Past Perfect ) .

Comme avec l'inaccompli la présence d'un auxiliaire signale la vision de l'énonciateur par rapport à un repère-point de vue, mais alors qu'avec l'inaccompli il y a identification entre le moment du procès et le moment repère avec l'accompli il $\mathrm{y}$ a différentiation entre ces deux moments : l'auxiliaire have, marqueur de localisation, construit le procès comme accompli au moment repère, la marque du participe passé (-en) témoigne de l'état résultant du procès et le présent ou le passé de la concomitance de cet état avec le moment repère.

Si le procès se situe bien antérieurement au moment d'énonciation, la vision qu'en a l'énonciateur ne donne plus à voir le procès lui-même qui n'est d'ailleurs pas localisé à l'aide de marques lexicales (ou autrement), mais l'état résultant de ce procès au moment repère. Le moment du procès $T_{E} n$ 'est pas connu car il n'est pas pertinent. La borne droite associée au procès est fermée pour symboliser qu'il est accompli. Seul compte l'état résultant dans la zone d'adjacence au procès et qui correspond à un intervalle ouvert à droite.

\section{Représentation topologique}

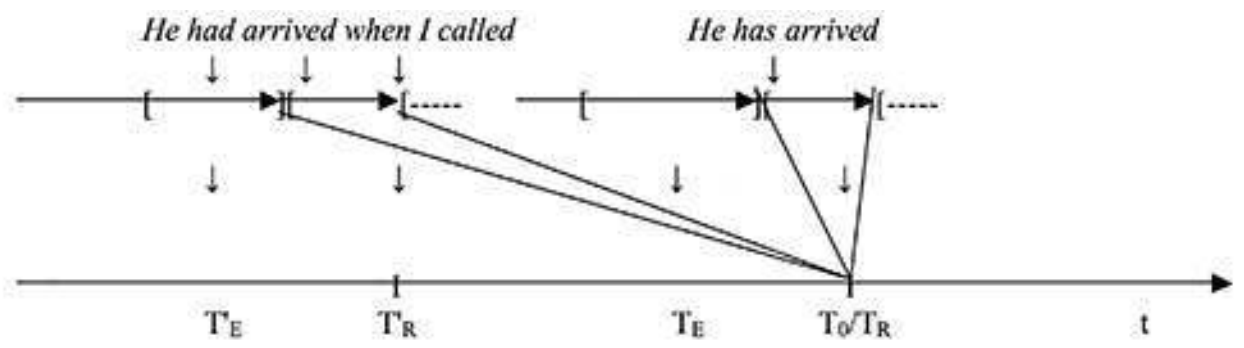

Dans le cas du présent l'auxiliaire have signale l'accomplissement du procès au moment d'énonciation $T_{0}$, qui est aussi le moment repère $T_{R}$ signalé par le présent, et le participe passé (-en) l'état résultant du procès tel qu'il est perçu à ce moment. On en déduit que le 
procès est situé à un moment antérieur $\mathrm{T}_{\mathrm{E}}$ non précisé (d'où l'absence de repère sur l'axe du temps).

Dans le cas du passé, la vision de l'énonciateur (la fenêtre) a toujours son origine au moment d'énonciation mais porte sur un procès dont le moment repère $T_{R}^{\prime}$ situé dans le passé est marqué explicitement ou implicitement. L'auxiliaire have marque toujours l'accomplissement du procès (qui a eu lieu à un moment indéterminé $\mathrm{T}_{\mathrm{E}}^{\prime}$ ) au moment repère $T_{R}^{\prime}$ signalé par le prétérit et le participe passé $(-e n)$ indique toujours l'état résultant du procès à ce moment repère.

\section{La composition d'aspects}

61 La forme have+be+V-ing (le fermé de l'ouvert) combine les caractéristiques des deux aspects. Tout inaccompli comportant une portion non nulle d'accompli, le procès donne à voir l'état résultant de cette portion d'accompli au moment repère. Elle ne peut concerner que des procès de type non terminatifs. Si l'énonciateur tient compte de l'activité du sujet (be+V-ing), il ne renvoie pas au processus lui-même mais au terme d'une partie du procès (have+en). Ce n'est pas le terme du procès qui est pris en compte (puisqu'il n'est pas forcément arrivé à son terme au moment repère) mais l'état résultant de la partie de procès prise en considération : c'est le type d'activité à laquelle s'est livré le sujet qui a pour conséquence un nouvel état du sujet. Ainsi l'intervalle ouvert à droite associé à cet état coïncide en partie avec l'ouvert associé au procès si celui-ci se poursuit au moment repère.

\section{Représentation topologique}

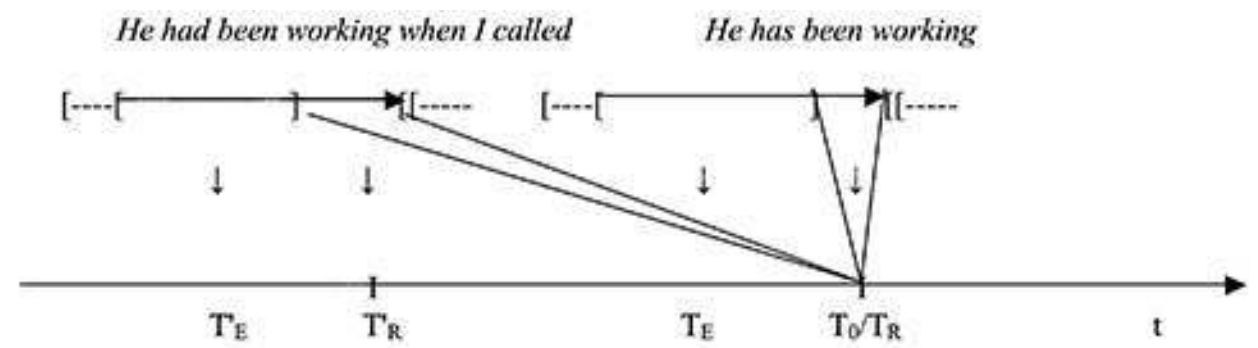

Au présent, l'auxiliaire have indique que la portion de procès considérée est accomplie au moment d'énonciation $T_{0}$, qui est aussi le moment repère $T_{R}$ signalé par le présent. La marque de l'inaccompli -ing correspond au processus pris en compte. Le participe passé (en) signale l'état résultant (marqué par la première borne droite ouverte) au moment repère de cette portion de procès dont la fin est marquée par la borne fermée à gauche des bornes droites ouvertes. Le procès peut toujours être en cours au moment d'énonciation (c'est pourquoi la borne à l'extrême droite est aussi ouverte). Si le procès est achevé en $T_{0} / T_{R}$, son moment $T_{E}$ reste indéterminé car il n'est pas pertinent.

$\mathrm{Au}$ passé, le moment repère $\mathrm{T}_{\mathrm{R}}^{\prime}$ est obligatoirement marqué de façon explicite ou implicite. L'auxiliaire have indique toujours que la portion de procès considérée est accomplie au moment repère signalé par le prétérit. La marque de l'inaccompli -ing indique le processus pris en compte et celle du participe passé (-en) l'état résultant (signalé par la première borne droite ouverte) au moment repère de cette portion de procès. La seconde borne droite est aussi ouverte car le procès pouvait toujours être en 
cours au moment repère. La borne fermée située à gauche des bornes droites ouvertes indique la fin de la portion de procès prise en compte. Si le procès était achevé au moment repère son moment $\mathrm{T}_{\mathrm{E}}^{\prime}$ reste indéterminé car non pertinent.

\section{Conclusion}

Nous avons vu au cours de cette étude que les temps et les aspects en anglais constituent un système de représentations multiples qui s'appuient sur une conception invariante du temps et qu'il est impossible de se limiter à l'étude des marqueurs linguistiques de surface mais qu'il est nécessaire de les relier aux opérations dont ils sont la trace et, au delà, à des représentations sémantico-cognitives ancrées dans la représentation perceptuelle du temps.

Après avoir rappelé comment la représentation cognitive du temps se faisait en partie sur le modèle de celle de l'espace, nous avons montré le lien entre cette représentation et les procédés linguistiques utilisés puis nous avons vu comment ces procédés étaient mis en oeuvre dans la langue. Cette étude a permis d'établir l'existence d'un continuum entre les multiples emplois du présent simple, de l'événement ponctuel au fait atemporel, et de voir comment ils sont uniformisés au prétérit. Nous avons vu aussi qu'un processus et un état résultant ont la même représentation topologique (des intervalles ouverts, qui se superposent partiellement dans le cas d'une combinaison d'aspects) et ne diffèrent qu'en termes de dynamicité.

Il ne s'agit là que d'une ébauche qui demande à être considérablement développée et élargie. Il reste notamment à rendre compte de l'emploi des temps dans les temps composés, dans les énoncés modalisés, dans les cas de repérage par rapport à une subordonnée (hypothétique, temporelle,...) ou dans des énoncés comme He's working everyday now. Nous n'avons pas abordé non plus l'influence des adverbes temporels sur la valeur de vérité des procès (Vlach, 1981) ni la temporalité intertextuelle (Chagnoux, 2006), qui est un autre sujet. Nous espérons néanmoins que cette étude aura permis de prendre la dimension du problème posé.

\section{BIBLIOGRAPHIE}

Asic, T. (2004). La représentation cognitive du temps et de l'espace : études pragmatiques des données linguistiques en français et dans d'autres langues. Thèse, Université Lumière-Lyon2.

Bybee, J., D. Slobin. (1982). « Rules and schemas in the Development and Use of the English past Tense ». Language, vol. 58, $\mathrm{n}^{\circ} 2$. pp. 265-289.

Chagnoux, M. (2006). Temporalité et aspectualité dans les textes français : modélisation sémantico-cogntive et traitement informatique. Thèse, Université Paris IV Sorbonne.

Col, G., B. Victorri. (2007). « Comment formaliser en linguistique cognitive ? Opération de fenêtrage et calcul du sens temporel ». CORELA, Cognition, Discours, Contexte. 
Croft, W., D. Cruise. (2004). Cognitive Linguistics. Cambridge, Cambridge University Press.

Culioli, A. (1999). « Les modalités d'expression de la temporalité sont-elles révélatrices de spécificités culturelles ». Pour une linguistique de l'énonciation, Tome 2. Paris, Ophrys. pp. 159-178

Desalles, J-L., L. Ghadakpour. (2004). In D. Badariotti (éd.), Le temps dans les systèmes naturels et artificiels, Actes des journées de Rochebrune -ENST 2004- S- 001, Paris.

Desclés, J-P. (1991). « Architectures, représentations cognitives et langage naturel ». In G. Vergaud (éd.), Les sciences cognitives en débat. Paris, CNRS. pp. 121-147.

Desclés, J-P. (1991). « La prédication opérée par les langues ». Langages, vol. 25. pp. 83-96.

Descles, J-P. (1993). « Un modèle cognitif d'analyse des temps du français ». Catégories grammaticales : temps et aspects, Séoul.

Descles, J-P. (1993). «Interaction entre langage, perception et action ». Faits de langues, n 1. pp. 124-127.

Descles, J-P. (1993). « Remarques sur la notion de processus inaccompli ». Sémiotiques, nº 5. Desclés, J-P. (1994). « Réflexions sur les Grammaires Cognitives ». Modèles Linguistiques.

Desclés, J-P. (1994). « Quelques concepts relatifs aux temps et à l'aspect pour l'analyse des textes ». Studia Kognitywne, Polska Akademia Nauk, Institut Slawistiki. pp. 57-88.

Desclés, J-P. (1998). « Les représentations cognitives du langage sont-elles universelles », in Essais sur le langage, logique et sens commun. Fribourg, Editions Universitaires. pp. 53-81.

Desclés, J-P., Z. Guenchéva. (1978). « Construction formelle de la catégorie grammaticale de l'aspect », Actes du colloque sur la notion d'aspect. Metz, Klincksieck. pp. 195-237.

Desclés, J-P., Z. Guenchéva. (2006). « Référentiels aspecto-temporels dans les textes ». Studia Kognitywne. Varsovie, Académie des Sciences de Pologne. pp. 11-38.

Desclés, J-P. (2010). « Reasoning in Natural Language in Using Combinatory Logic and Topology: an Example with Aspect and Temporal Relations ». Proceedings of the $23^{\text {rd }}$ International Florida Artificial Intelligence Research Society Conference, FLAIRS. pp. 174-179.

Evans, V. (2001). « Why does time flow and where does it flow to? The temporal matrix and conceptual blending ", http://www.cogs.susx.ac.uk/users/vyv.

Fauconnier, G., M .Turner. (2008). The Way we Think. New-York, Basic Books.

Fauconnier, G., M. Turner. (1999). «A Mechanism of Creativity », Poetics Today,Vol.20, n 3. pp. 397-418.

Fauconnier, G., E. Sweetser. (1996). Spaces, Worlds and Grammar. Chicago, The University of Chicago Press.

Fuchs, C. (2004). La Linguistique Cognitive. Gap, Ophrys.

Givon, T. (1973). « The time axis phenomenon », Language, vol. 49, n 4, pp. 890-925.

Givon, T. (1993). English Grammar, vol. 1. Amsterdam/Philadelphia, John Benjamins.

Givon, T. (2001). Syntax vol. 1. Amsterdam/Philadelphia, John Benjamins.

Harper, M.P., E. Chamiak (1986). « Time and Tense in English ». Proceedings of the $24^{\text {th }}$ annual meeting of the Association for Computational Linguistics. New-York. pp. 3-9. 
Guentchéva, Z. (2010). « Référentiels aspecto-temporels ». L'analisi linguistica e letteraria, XVIII. pp. 69-80.

Heine, B. (1997). Cognitive Foundations of Grammar. Oxford, Oxford University Press. Hornstein, N. (1977). « Towards a theory of tense ». Linguistic Inquiry, vol. 8, N 3. pp. 521-557. Jackendoff, R. (1985). Semantics and cognition. Cambridge (Mass.), The MIT Press. Jackendoff, R. (1993). Patterns in the Mind. New-York, Harvester Wheatsheaf. Jackendoff, R. (1991). « The problem of Reality ». Noûs, vol. 25, nº 4. pp. 411-433. Johnson, M. (1990). The body in the Mind. Chicago, The University of Chicago Press. Joly, A., D. O'Kelly. (1991). Grammaire Systématique de l'Anglais. Paris, Nathan. Lakoff, G., M. Johnson. (1980). Metaphors We Live By. Chicago, Chicago University Press. Lakoff, G., M. Johnson. (1980). Conceptual metaphor in everyday language. The Journal of Philosophy, vol. 77, $\mathrm{n}^{\circ} 8$.

Langacker, R. (1991). Concept, Image and Symbol. Berlin / New-York, Mouton de Gruyter. Langacker, R. (2000). Grammar and Conceptualization. New-York, Mouton de Gruyter. Langacker, R. (2008). Cognitive Grammar. Oxford, Oxford University Press. Lapaire, J-R, (2006). « Réification, ception, cognition (1/2)». Bulletin de la Société de Stylistique Anglaise, $n^{\circ} 28$, p. 25-53.

Michon C. (1992). «Introduction». In Macar, F. Pouthas V.\& Friedman W. (eds.), Time, Action and Cognition: Towards Bridging the Gap. Dordrecht, Kluwer. pp. 2-20.

Pankhurst, N.J. (1980). « Closer to a theory of tense for contrastive analysis ». PSCL12. 115-136 Radden, G., R. Dirven. (2007). Cognitive English Grammar. Amsterdam, John Benjamins. Reichenbach, H. (1947). Elements of Symbolic Logic. New-York, Free press. Saussure, L. de. (2003). Temps et pertinence : éléments de pragmatique cognitive du temps, Bruxelles, Duculot.

Talmy, L. (2000). Toward a Cognitive Semantics, vol. 1. Cambridge (Mass.), The MIT Press.

Taylor, J. (1996). Cognitive Grammar. New-York/Oxford, Oxford University Press.

Taylor, J. (2003). Linguistic Categorization. New-York/Oxford, Oxford University Press.

Turner, M. (1990). « Poetry: Metaphor and the Conceptual Context of Invention ». Poetics Today, vol. 11, n 3. pp. 463-482.

Turner, M. (1996). The Literary Mind. New-York/Oxford, Oxford University Press.

Van de Velde, D. (2006). Grammaire des événements. Villeuneuve d'Asq, Presses Universitaires du Septentrion.

Vendler, Z. (1957). « Verbs and Times ». The Philosophical Review, vol. 66, No. 2. pp. 143-160.

Vlach, F. et F. Nef. (1981). « La sémantique du temps et de l'aspect en anglais ». Langages, $\mathrm{n}^{\circ} 64$. pp. 65-79.

Yip, K. M. (1985). « Tense, Aspect and the Cognitive Representation of Time ». IJCAI Proceedings. pp. 806-814. 


\section{NOTES}

1. On dit que tel endroit est "à 2 heures de marche/train/avion". Les astrophysiciens mesurent en années-lumière.

2. Par exemple, pour les indiens Hopi d'Amérique du Nord le temps est un présent qui s'étend indéfiniment.

3. Le moment d'énonciation est abusivement représenté de façon ponctuelle pour des raisons computationnelles.

4. A la suite de Louis de Saussure (2003, p. 30) nous dirons que les distinctions dans la typologie des procès sont $d$ 'ordre conceptuel et non ontologique, la perception du réel passant par le filtre de l'interprétation mentale.

5. "Verbe de processus" désigne ici l'aspect lexical du verbe par opposition à "procès de type processus" qui fait référence à l'aspect grammatical.

6. L'adjectif "concomitant" est préférable à "coïncident" car ce dernier est généralement employé pour signifier que le procès est repéré en identification par rapport au moment d'énonciation.

7. Cette différence est parfois gommée quand elle n'est pas pertinente, comme dans les indications scéniques où les auteurs emploient souvent indifféremment les deux formes.

8. Sauf si le procès fait aussi l'objet d'un commentaire de l'énonciateur : I'm warning you.

9. C'est ainsi que dans In 1750, Bach dies la marque temporelle antéposée concerne seulement l'occurrence de l'événement, pas son existence qui est, elle, atemporelle.

10. Sauf dans certains cas particuliers : You wouldn't say she was forty ("backshifting"), I wanted to ask you ("tentative use"). De même avec be+V-ing (voir 6. ci-dessous): Originally, I was leaving tomorrow.

11. Les localisateurs doivent cependant être compatibles avec la sémantèse du verbe : on ne pourra dire I liked chocolate *at two o'clock/*this morn ng/ ?yesterday.

12. On assimile ici les procès réitérés par l'adjonction d'une marque lexicale (everyday) aux procès itératifs (hop).

\section{RÉSUMÉS}

L'objectif du présent article est d'essayer de rapporter dans le domaine de la temporalité les opérations linguistiques aux opérations cognitives qui les sous-tendent. Après avoir établi le lien entre représentation spatiale et temporelle l'article examine la façon dont le temps est conceptualisé puis représenté dans la langue. Il applique ensuite les résultats à l'étude des temps simples et des aspects en anglais contemporain.

The aim of this article is to link the linguistic operations in the expression of time to the underlying cognitive operations. After establishing a link between spatial and temporal representation it examines the way in which time is conceptualized and represented in language. It then applies its findings to the study of the simple tenses and aspects of English. 
INDEX

Mots-clés : opérations, représentation, conceptualisation, aoriste, accompli, inaccompli, événement, état, fait

Keywords : conceptualization, aorist, accomplished, unaccomplished, event, state, fact

\section{AUTEUR}

\section{GILBERT GHIO}

Université de Toulon 